\title{
Electric field inversion asymmetry: Rashba and Stark effects for holes in resonant tunneling devices
}

\author{
H. B. de Carvalho, ${ }^{1}$ M. J. S. P. Brasil, ${ }^{1}$ V. Lopez-Richard, ${ }^{2, \text { ศ }}$ I. Camps,${ }^{3}$ Y. Galvão \\ Gobato,,${ }^{3,}$ G. E. Marques, ${ }^{3}$ L. C. O. Dacal,${ }^{4}$ M. Henini,${ }^{5}$ L. Eaves,${ }^{5}$ and G. Hill ${ }^{6}$ \\ ${ }^{1}$ Grupo de Propriedades Ópticas, Instituto de Física Gleb Wataghin, \\ Universidade de Campinas, 13083-970, Campinas, SP, Brazil. \\ ${ }^{2}$ Faculdade de Filosofia Ciências e Letras de Ribeirão Preto, Departamento de Física e Matemática, \\ Universidade de São Paulo,14040-901, Ribeirão Preto, SP Brazil. \\ ${ }^{3}$ Universidade Federal de São Carlos, Departamento de Física, 13560-905, São Carlos, SP, Brazil. \\ ${ }^{4}$ Instituto de Estudos Avançados, IEAv - CTA, C. P. 6044, 12231-970, São José dos Campos, SP, Brazil. \\ ${ }^{5}$ School of Physics and Astronomy, University of Nottingham, NG7 2RD, Nottingham, UK \\ ${ }^{6}$ EPSRC National Centre for III-V Technologies, \\ University of Sheffield, Mappin Street, S1 3JD, Sheffield, UK
}

(Dated: June 26, 2018)

\begin{abstract}
We report experimental evidence of excitonic spin-splitting, in addition to the conventional Zeeman effect, produced by a combination of the Rashba spin-orbit interaction, Stark shift and charge screening. The electric-field-induced modulation of the spin-splitting are studied during the charging and discharging processes of p-type GaAs/AlAs double barrier resonant tunneling diodes (RTD) under applied bias and magnetic field. The abrupt changes in the photoluminescence, with the applied bias, provide information of the charge accumulation effects on the device.
\end{abstract}

The effect of the spin-orbit (SO) interaction in quasitwo-dimensional (Q2D) systems has attracted renewed attention in recent years. The topic has been on the focus of many optical and transport investigations of spin-related phenomena in nanoscopic systems [1, 2, 3], a subject of great fundamental and technological interest [4, 5, 6, 7]. In this letter, we address experimental evidence of electric field coupling to the spin degree of freedom of carriers in RTD; here in particular, the prevailing influence can be attributed to the $\mathrm{SO}$ and Stark effects on the hole electronic structure. These interactions are relevant to the study of the internal electric fields and the charge accumulation in the structure. The simultaneous investigation of optical and transport properties at high magnetic and electric parallel fields, has permitted a thorough characterization of the main processes involved in the system response. The novelty of this result consists of the optical detection of electric field modulation of the effective spin-splitting beyond the Zeeman effect and its unambiguous correlation to the transport mechanisms which is responsible for the charge buildup in the states of the RTD.

This study is carried out on a symmetric $p-i-p$ GaAs/AlAs RTD, that has been previously used to characterize hole space charge buildup and resonant effects in a magnetic field [8]. The structure is in the form of a $400 \mu \mathrm{m}$ diameter mesa with a metallic $A u G e$ annular top contact to allow optical access. The diode was mounted in a superconducting magnet and the emission spectra were recorded using a double spectrometer coupled to a CCD system with polarizer facilities to select left (right) $\sigma^{+(-)}$configurations. When light from an $\mathrm{Ar}^{+}$laser is focused close to the surface, minority electrons are created [8]. As the bias approaches a resonant condition, the carrier density inside the QW increases and then decreases, resulting in the negative differential resistance (NDR) region when the resonance is traversed. The photo-generated electrons tunneling into the $\mathrm{QW}$ layer can recombine with the injected holes or tunnel out of the well layer. These processes are represented schematically in the Fig. 1(a).

The $I-V$ characteristics, shown in Fig. 1(b), displays a series of peaks associated with the injected holes $\left(I_{\text {dark }}\right)$ from the hole accumulation layer formed in the outside interface of the diode (see Fig. 1 (a)). Under illumination, an increase of current is observed $\left(I_{\text {light }}\right)$ due to the injection of minority electrons. Under this condition, is noted the emergence of polarized photoluminescence $(\mathrm{PL})$ emissions from electron-hole (e-h) pair recombinations inside the QW layer, as shown schematically in the top panel. These PL spectra were recorded for $\sigma^{+}$and $\sigma^{-}$polarizations as a function of the bias. The energy of the PL peaks as well as the resulting spin-splitting as a function of bias are shown in Figs. 1 (c) and (d), respectively. We now focus on the mechanisms responsible for the abrupt changes detected at the critical voltages, marked with dashed-lines, in Fig. 1 As the bias (electric field) increases, both PL polarized peak energies show the expected red-shift induced by the quantum Stark effect, but also the sharp discontinuities at the hole-resonant voltages. The abrupt variation of the PL-peak energies has been attributed to changes in the QW level charge density near the critical voltages; thus inducing changes on the built-in electric field [9]. These discontinuities are more evident in the total spin-splitting energy vs bias, shown in Fig. 10(d).

Fig. 1 (d) shows fluctuations of the spin-splitting energy and an increasing trend as bias is swept between 


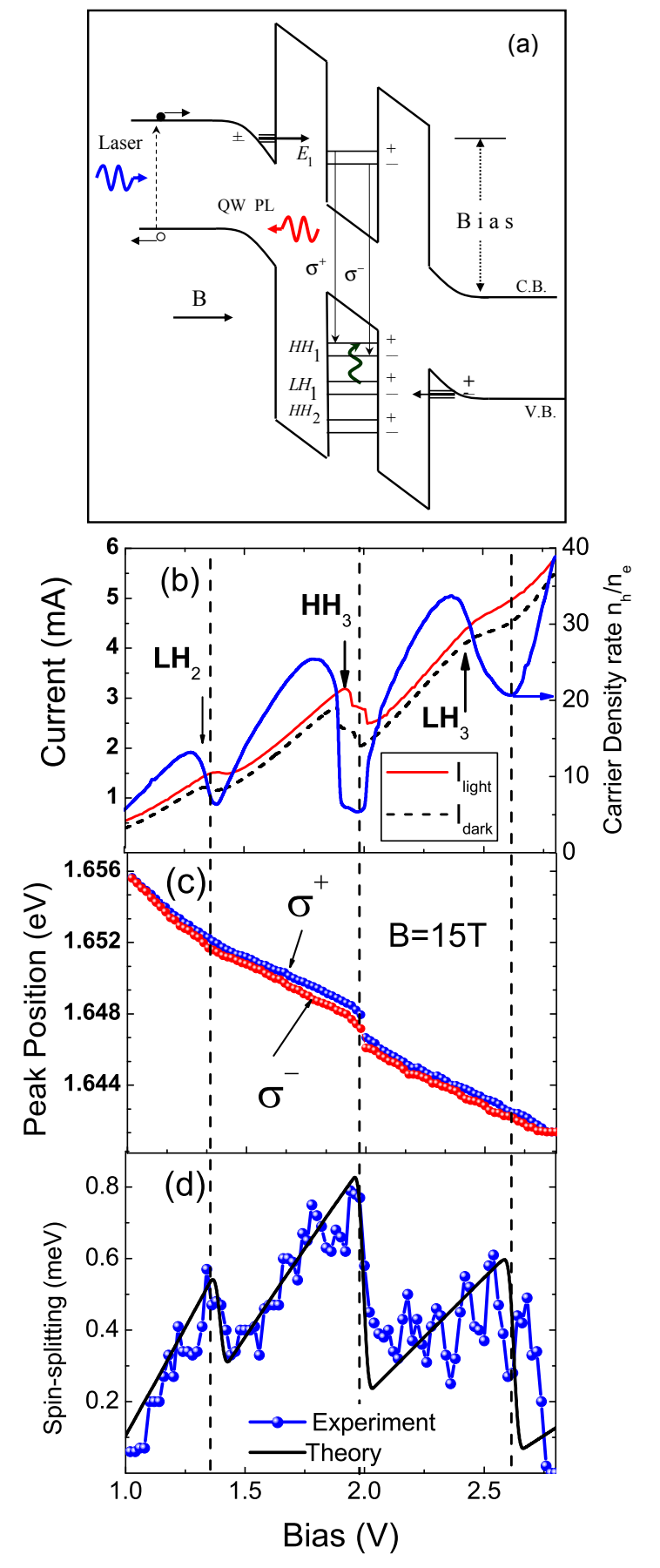

FIG. 1: Panel (a): Schematic diagrams for tunneling and recombination processes in the GaAs - AlAs RTD with $4.2 \mathrm{~nm}$ (5.1nm) well (barriers) width, under illumination. Below panels: Vertical dashed-lines show the critical voltages where abrupt changes in the properties occur for $B=15 \mathrm{~T}$. Panel (b): The $I-V$ characteristics with $\left(I_{\text {light }}\right)$ and without $\left(I_{\text {dark }}\right)$ illumination. The carrier density rate calculated through Eq. (3). Panel (c): Energy peak positions of $\sigma^{ \pm}$PL spectra as a function of bias. Panel (d) Symbols: measured spin-splitting of excitonic recombinations, for increasing bias. Solid-line: Calculated spin-splitting generated by SO effects, in addition to the conventional Zeeman effect. two hole critical voltages. The sharp decrease occurs when the NDR region is crossed and the QW level is being discharged. Besides the usual Zeeman effect, other two SO interactions modify the effective spin-splitting: (i) the bulk inversion asymmetry (BIA or Dresselhaus $\mathrm{SO}$ ) induces spin-splitting only in zincblende heterostructures, (ii) the structure inversion asymmetry (SIA or Rashba SO) causes spin-splitting under applied electric field [10, 11]. Both contributions depend on the material SO parameters and their effects can be enhanced by the presence of a magnetic field [10]. The properties addressed in this work occur at high electric field (bias), then the Rashba SO contribution becomes dominant and we will not consider the BIA SO term.

The complex behavior of our device is studied by modeling the states by a square potential with a bias dependent electric field due to the charge density built in the QW levels. The coupling between this effective electric field and the spin degree of freedom is introduced via the Rashba SO Hamiltonian for electrons 10, 11] and for holes 12. The kinetic Luttinger Hamiltonian provides an accurate description of the valence band admixture and permits to treat magnetic $(B)$ and electric $(F)$ fields, as well as the Rashba SO in the same framework. The full Hamiltonian, $H^{\text {cond } / v a l}=H^{L} \pm I_{2 j+1} \cdot e F z+H^{S O}$, may be decomposed into three terms: (i) $H^{L}$ that describes the dynamics associated with the Landau and Zeeman quantization in a magnetic field, (ii) $I_{2 j+1} \cdot e F z$ contains all Stark shift-related terms that produce the inversion asymmetry induced by the electric field $(F)$; here $I_{2 j+1}$ is the $(2 j+1)$-rank unity matrix, and (iii) the Rashba SO term that couples the dynamical linear momentum with the spin degree of freedom. The SO term is treated in the $(2 j+1)$-rank representation of the total momentum with $j=1 / 2$ for electrons and $j=3 / 2$ for holes 12 as

$$
H_{\gamma}^{S O}=\alpha_{\gamma} \frac{\sqrt{2} F}{\lambda_{c}} i\left(a J_{+}-a^{\dagger} J_{-}\right)
$$

Here $\alpha_{\text {cond(val) }}$ is the Rashba SO parameter for conduction (valence) band, $\lambda_{c}$ is the magnetic cyclotron radius, and $J_{ \pm}=\frac{1}{2}\left(J_{x} \pm J_{y}\right)$, where $J_{i}$ is the $4 \times 4(2 \times 2)$ angular momentum matrix for holes (electrons). One advantage of this $\mathrm{SO}$ representation is that it permits a wavefunction expansion in each component of the spinor state with the required symmetries defined by the lateral and vertical confinement types. The basis set also combines the band edge periodic Bloch functions in the total momentum representation: $|s \uparrow \downarrow\rangle,|h h \uparrow \downarrow\rangle,|l h \uparrow \downarrow\rangle$, the vertical eigenstates: $A_{2 k-1}(z)$-even and $A_{2 k}(z)$-odd parity for $k=1,2, \ldots$; and the lateral Landau states $|N\rangle$. The eigenfunctions for the conduction $\left(\Phi_{c}\right)$ and for the 
valence band states $\left(\Phi_{v}\right)$ have the general form,

$$
\Phi_{c}=\left[\begin{array}{c}
A_{1}^{c}|N\rangle|s \uparrow\rangle \\
A_{1}^{c}|N+1\rangle|s \downarrow\rangle \\
A_{2}^{c}|N\rangle|s \uparrow\rangle \\
A_{2}^{c}|N+1\rangle|s \downarrow\rangle \\
A_{3}^{c} \ldots
\end{array}\right], \Phi_{v}=\left[\begin{array}{c}
A_{1}^{v}|N-2\rangle|h h \uparrow\rangle \\
A_{1}^{v}|N-1\rangle|l h \uparrow\rangle \\
A_{1}^{v}|N\rangle|l h \downarrow\rangle \\
A_{1}^{v}|N+1\rangle|h h \downarrow\rangle \\
A_{2}^{v}|N-2\rangle|h h \uparrow\rangle \\
A_{2}^{v}|N-1\rangle|l h \uparrow\rangle \\
A_{2}^{v}|N\rangle|l h \downarrow\rangle \\
A_{2}^{v}|N+1\rangle|l h \downarrow\rangle \\
A_{3}^{v} \ldots
\end{array}\right] .
$$

Note that $N=-1,0,1,2, \ldots$ is an effective Landau index labeling the lateral confinement symmetries (parities) of each component. The sequence of periodic valence Bloch states in the components is determined by the sequence chosen to write the Luttinger Hamiltonian. These vector states have, in principle, infinite dimension since the index $k$, used to enumerate the vertical functions $A_{2 k-1}$ and $A_{2 k}$, runs over all positive integer numbers.

Fig. 2] (a) shows the calculated electron and hole magnetic dispersions for the lowest energy subband of carriers in the conduction $\left(E_{1}\right)$ and in the valence $\left(H H_{1}\right.$, $L H_{1}$ ) bands of the GaAs QW. Note that the effective Zeeman splitting for holes and electrons can be tuned by the external fields $F$ and $B$. This also reflects the strong admixture of states generated by the combination of Rashba SO plus Stark effects, in particular, for the valence band where the strong nonparabolicity is present in the kinetic Luttinger Hamiltonian. A comparison between the contribution from the nonparabolicity $(F=0)$ and from SO plus Stark effects $(F=100 \mathrm{kV} / \mathrm{cm})$ to the effective spin-splitting of the $H H_{1}$ ground state, is shown in Fig. 2 (b) for different degrees of vertical confinement. For fixed $B$-value, an increase of well width increases the energy splitting since the coupling between states with close energy is enhanced. The corresponding interband transition energies, calculated for a GaAs QW, are displayed in Fig. 22 (c) as a function of the electric field. The inversion asymmetry, introduced by the electric field, effectively couples QW subbands with different lateral and vertical symmetries. This coupling leads to the spin-splitting modulation by the electric field, which is the main topic addressed. Note that the strength of modulation can be enhanced for materials with larger SO parameters as shown in Fig. 2] (d). The larger is the Rashba SO parameter, such as in InAs or InSb, the stronger is the coupling between the electric field and the spin-degree of freedom.

Superimposed to the external electric field (bias), there is an internal field component due to the charge distribution throughout the diode structure. The relation between the applied bias and the total effective electric field $F$ experienced by the carriers would require a selfconsistent calculation. Here we consider an average effective field which qualitatively accounts for the main effects of

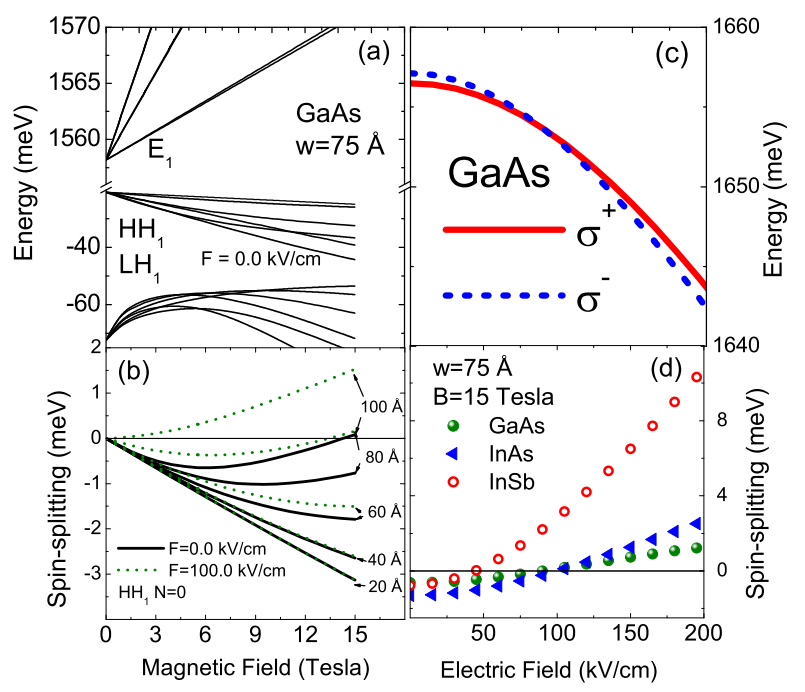

FIG. 2: Panel (a): Landau level fan charts $(N=0,1,2)$ for the first electron $\left(E_{1}\right)$ and hole $\left(H H_{1}, L H_{1}\right)$ subbands in a GaAs QW with width $w=75 \AA$ and $F=0$. Panel (b): Calculated Zeeman splitting of the $H_{1}$ ground state. The solid-lines (dashed-lines) indicate the results without (with) the inversion asymmetry induced by the electric field. Panel (c): Interband transition energies for $\sigma^{ \pm}$polarized light emissions in a GaAs QW, with $B=15$ Tesla. Panel (d): The additional SO contribution to the calculated spin-splitting energy of excitonic recombination in identical QW's of different III-V compounds, as a function of the electric field.

the inversion asymmetry on the spin-splitting energy seen in the PL emissions. From $I-V$ characteristics with and without illumination [see Fig. [1(a)] we may estimate the ratio between the majority $\left(n_{h}\right)$ and minority $\left(n_{e}\right)$ carrier densities inside the $\mathrm{QW}$, as

$$
\frac{n_{h}}{n_{e}}=2 \frac{I_{\text {dark }}(V)}{I_{\text {light }}(V)-I_{\text {dark }}(V)} .
$$

Together with the $I-V$ characteristics, this ratio is shown in panel (b) of Fig. 1, as a function of bias and for the highest laser excitation power. Note that in our experimental conditions the density of holes (majority) is always larger than the density of electrons (minority). Moreover, $n_{h} / n_{e}$ increases smoothly during the charge buildup process, as the bias is being swept between two hole resonant voltages and an abrupt drop occurs during the QW discharge (NDR region).

Owing to the smooth variation of $n_{h} / n_{e}$ and to the experimental condition $n_{h} \gg n_{e}$, we have chosen to use a total field $F$ changing linearly with an effective charge density, $n_{h}^{e f f}$, and giving rise to an uniform field. In the presence of an asymmetry induced by the external field, this is a fair approximation [13, 14, 15], and can be written

$$
F=F^{e x t}+\frac{e}{\epsilon} n_{h}^{e f f}
$$


where $\epsilon=13.18$ is the static dielectric constant for $\mathrm{GaAs}$. All the other parameters for $G a A s$ used in the simulation, such as effective masses, Luttinger and Rashba parameters were taken from the literature.

Based on these assumptions we have estimated the asymmetry effects on the modulation of spin-splitting seen in the PL emissions, due to the charge accumulation in the QW levels. This is compared with experimental results in Fig. 10 (d). A percentage of the experimental wiggly increase is due to charge fluctuations on system. Our theoretical model does not take into account any type of charge fluctuations.

For this Q2D hole-rich gas, the PL emissions must arise from the recombination of either positive trions $\left(X^{+}\right)$or neutral excitons $\left(X^{0}\right)[16,17]$. In order to understand the behavior of the PL peaks in Fig. 1 (c) with abrupt variations at resonant transitions for both spin-up and spin-down recombinations, we must consider the charge density-dependent screening on the binding energy of the excitonic complexes involved in the PL emissions [18]. An increase in the Q2D carrier density leads to screening of the Coulomb interaction that inevitably weakens the binding energy of the excitonic complexes [18]. Moreover, the charge buildup also gives rise to modulations to: (i) the e-h Coulomb interaction and; (ii) the Stark shift. Cases (i) and (ii) give opposite contributions to the optical transition energies. Near the critical voltages, case (ii) produces a blue-shift due to the reduction of the effective electric field, whereas case (i) induces a red-shift due to the decrease of the excitonic binding energies. These competing effects give origin to the abrupt changes in the peak position of $\sigma^{+}$and $\sigma^{-}$PL emissions of Fig. 1 (c). Therefore, the spin-splitting energy is not affected by these excitonic corrections.

As final comments, the good agreement with experimental increasing trend required an effective density in the range $6 \cdot 10^{12} \mathrm{~cm}^{-2}<n_{h}^{\text {eff }}<14 \cdot 10^{12} \mathrm{~cm}^{-2}$. This is larger than the values reported in Ref. [8] using a different relation between bias and local fields. The larger value for the effective hole charge density is due to the neglected contribution coming from the hole density in interface accumulation layer. Furthermore, the effective QW width $w=75 \AA$ was adjusted to fit the $e-h$ pair recombination energy at $F=0$. In this $p-i-p$ sample, the modulation of the excitonic spin-splitting is strongly related to valence band admixture. As noted in Fig. 2 (a), the set of $H H_{1}$ and $L H_{1}$ Landau dispersions are highly nonparabolic, in contrast to the almost parabolic conduction band levels. This effect is strongly affected by the QW width that determines the separation between the coupled $H H_{1}$ and $L H_{1}$ subbands, as shown in Fig. 2 (b).

In summary, we have observed that spin-splitting dependence on bias is mainly influenced by the renormalization of the hole charge density in the uniform field approximation. This spin-splitting induced by the combined effects of Rashba SO interaction, Stark effect, Zee- man and interband couplings acts as a probe of the relation between the fluctuating internal field $F$ and the continuously varying bias $V$. We have shown how the electronic structure is affected by the modification of the effective field $F$ in our RTD and by the modulation of the Rashba SO and screening effects induced by charge fluctuations. We have studied these effects in simultaneous optical and transport measurements of the main physical processes involved.

The authors acknowledge financial support from Brazilian agencies FAPESP and CNPq and from the UK Engineering and Physical Sciences Research Council.

* Electronic address: vlopez@df.ufscar.br

$\dagger$ also at Departamento de Física, Universidade Federal de Santa Catarina.

[1] E. G. Mishchenko, A. V. Shytov, B. I. Halperin, Phys. Rev. Lett. 93, 226602 (2004).

[2] S.A. Tarasenko, V.I. Perel', I.N. Yassievich, Phys. Rev. Lett. 93, 056601 (2004).

[3] S.D. Ganichev, V.V. Bel'kov, L.E. Golub, E.L. Ivchenko, P. Schneider, S. Giglberger, J. Eroms, J. De Boeck, G. Borghs, W. Wegscheider, D. Weiss, W. Prettl, Phys. Rev. Lett. 92, 256601 (2004).

[4] D. Stepanenko, N. E. Bonesteel, Phys. Rev. Lett. 93, 140501 (2004).

[5] Y. Kato, R. C. Myers, A. C. Gossard, D. D. Awschalom, NATURE 427, 50 (2004).

[6] E.I. Rashba, A.L. Efros, Phys. Rev. Lett. 91, 126405 (2003).

[7] T. Koga, J. Nitta, H. Takayanagi, Phys. Rev. Lett. 88, 126601 (2002).

[8] R.K. Hayden, L. Eaves, M. Henini, D.K. Maude and J.C. Portal, G. Hill, Appl. Phys. Lett. 60, 1474 (1992).

[9] T.A. Fisher, P.D. Buckle, P.E. Simmonds, R. J. Teissier, S. Skolnick, C.R.H. White, D.M. Whittaker, L. Eaves, B. Usher, P.C. Kemeny, R. Grey, G. Hill, and M.A. Pate, Phys. Rev. B 50, 18469 (1994).

[10] Y.A. Bychkov, E.I. Rashba, J. Phys. C: Solid State Physics 17, 6039 (1984).

[11] G.E. Marques, A.C. Bittencourt, C.F. Destefani, and S.E. Ulloa, Phys. Rev. B 72, 045313, (2005).

[12] Marco G. Pala, Michele Governale, Jürgen König, Ulrich Zülicke, and Giuseppe Iannaccone, Phys. Rev. B 69, 045304 (2004).

[13] F. Stern, S. Das Sarma, Phys. Rev. B 30840 (1984).

[14] R. Winkler, H. Nohb, E. Tutucb, and M. Shayeganb; Physica E 12, 428 (2002).

[15] S.M. Landi, C.V.-B. Tribuzy, P.L. Souza, R. Butendeich, A.C. Bittencourt, and G.E. Marques, Phys. Rev. B 67, 085304 (2003).

[16] A. Vercik, Y. Galvão Gobato, I. Camps, G.E. Marques, M.J.S.P. Brasil, and S.S. Makler, Phys. Rev. B 71, 075310 (2005).

[17] F.J. Teran, L. Eaves, L. Mansouri, H. Buhmann, D.K. Maude, M. Potemski, M. Henini, and G. Hill Phys. Rev. B 71, 161309R (2005).

[18] L.C.O. Dacal and J.A. Brum, Phys. Rev. B 65, 115324 (2002). Also, J.A. Brum, G. Bastard, and C. Guillemot, 
Phys. Rev. B 30, 905 (1984). L.C.O. Dacal and J.A.

Brum, Physica E 12546 (2002). 